\title{
Ultrasonogram characteristics of ovarian thecoma
}

\author{
PAN Yu-ping ${ }^{1,2, a}$ \\ ${ }^{1}$ Medical College, Eastern Liaoning University, Dandong , Liaoning118003 China \\ ${ }^{2}$ Department of Ultrasound, Shengjing Hospital of China Medical University, Shenyang \\ ,Liaoning110004 China \\ aemail :Panxy900@sina.com
}

\begin{abstract}
Keywords: Ovary, Thecoma, Ultrasonography
Abstract: Objective To analyze the ultrasonogram characteristics of ovarian thecoma in order to improve the diganostic level for this disease. Methods The ultrasonic characteristics of 64 patients with ovarian thecoma confirmed with pathology were retrospectively analyzed.Results The tumors located at left side $(n=33)$, right side $(n=29)$, bilateral $(n=2)$; solid lesion beside uterus was found in 56 patients, mixed cystic-solid lesion in 5 and cystic lesion in 1 patient, 2 patients with ovarian thecoma were not detected by ultrasonography. The ultrasonic characteristics of all the 62 patients with ovarian thecoma were almost consistent with the pathologic characteristics.Conclusion Ovarian thecoma has certain ultrasnoc characteristics, the final diagnosis usually depends on pathology.
\end{abstract}

\section{Introduction}

This paper put forward ultrasnoc characteristics and differential diagnosis through retrospectively analyzed the ultrasonic manifestations of 64 patients with ovarian thecoma confirmed with pathology, in order to improve the diagnostic level of the disease.

\section{Clinical data and Methods}

\section{Clinical data}

64 patients of hospitalizationin and ovarian thecoma confirmed with pathology in Shengjing Hospital of China Medical University, age $19 \sim 76$ years, with a average age of $(52.5 \pm 11.9)$ years old.

\section{Methods}

TOSHIBA aplio XG、ACCUVTX XQ and Philips iU22 was used, married with transvaginal ultrasound combined with transabdominal ultrasound, with vaginal probe frequency of $5 \sim 9 \mathrm{MHz}$, unmarried by transabdominal ultrasonography, with probe frequency of $3 \sim 5 \mathrm{MHz}$, abdominal examination, bladder of patients was moderate filled, supine position; transvaginal examination, ask the patient to empty the bladder, dorsal lithotomy position, supine on the examination bed, vaginal probe surface coated with the coupling agent coating with the condom into the vagina of the patient; multi angle, multi direction, multi section scanning of uterus and bilateral annex.

Statistical analysis

SPSS17.0 software was used for statistical analysis.

\section{Results}

\section{The ultrasonographic manifestations of ovarian thecoma}

ultrasound detected 62 cases, 56 solid lesion, mixed cystic-solid lesion in 5 and cystic lesion in 1 patient, 2 patients with ovarian thecoma were not detected by ultrasonography, tumors showed inhomogeneous low echo in 28 cases, low echo in 24 cases, cystic-solid mixed echo in 5 cases, medium- low mixed echo in 4 cases, cystic echo in 1 cases, clear boundary in 46 cases, boundary less clear in 9 cases, fuzzy boundaries in 7 cases, shape is irregular in 18 cases, posterior echo with attenuation in 8 cases, blood flow can be detected in 17 cases by CDFI. 


\section{Serum CA125 examination}

9 cases with CA125 more than 2 times higher than normal.

\section{The age distribution of ovarian thecoma}

1 cases of 10-20 years, 20-30 years 2 cases, 7 cases of 31-40 years, 41-50 years 18 cases, 51-60 years 23cases, 8 cases of 61-70 years, 71-80 years 5 cases.

\section{Ovarian thecoma operation findings}

The ultrasonic characteristics of all the 62 patients with ovarian thecoma were almost consistent with gross morphology of seeing tumors in operation, but 2 patients with ovarian thecoma were not detected by ultrasonography, discovered in operation exploration, left ovary of one case is $4.0 \mathrm{~cm} \times 4.0$ $\mathrm{cm} \times 3.0 \mathrm{~cm}$, left ovary of one case showed diameter about $1.5 \mathrm{~cm}$ solid nodule, color white, hard, minimum thecoma $1.0 \mathrm{~cm}$ x $1.0 \mathrm{~cm}$, the largest thecoma $20.0 \mathrm{~cm}$ x $30.0 \mathrm{~cm}$;ovarian thecoma with 45 cases of pelvic effusion.

\section{Ovarian thecoma pathological results}

pathological return ovarian thecoma in 53 cases, thecofibroma in 11 cases, left thecoma in 33 cases, 29 cases of right thecoma, 2 cases of bilateral, including 8 cases of cell growth activity, simple ovarian thecoma in 24 cases,

26 cases of thecoma with simple myoma of uterus, 2 cases of endometrial polyp, 4 cases of adenomyosis, 3 cases of ovarian tumors , cervical CIN II 1 cases, 4 cases of mesenteric cyst of ovary and fallopian tube.

\section{Discussion}

On ovarian thecoma from ovarian sex cord stromal tissue, a type of thecofibroma group ${ }^{[1]}$, accounts for $0.5 \%-1 \%$ of ovarian tumors ${ }^{[2]}$, When thecoma aging internal fibrous tissue increased and formed thecofibroma $^{[3]}$, mainly occurs after menopause, over 45 years old accounts for $81.25 \%(52 / 64)$ in this paper, thecoma is functional tumors of ovary, caused endometrial hyperplasia of some patients with increased estrogen levels, some patients can appear pleural effusion, ascites, namely Meigs syndrome and CA125 rise, ascites is the characteristic of ovarian fibroma, very rare in other benign ovarian tumors ${ }^{[4]}$. In this paper patients of $70.31 \%$ (45/64) with pelvic cavity effusion,thus the ascites is not unique of ovarian fibroma, rise of serum CA125 is associated with production of ascites. Thecoma is unilateral, bilateral occur rarely, this group had 62 cases of unilateral, only 2 cases were bilateral .

\section{Ovarian thecoma sonographic characteristics}

( 1 ) Form were usually round, oval or lobulated, irregular; ( 2 ) Tumor has fibrous capsule, clear boundary, size is not fixed, small ovarian thecoma showed normal size, pathological findings after operation, big can occupy the whole pelvic cavity; ( 3 ) When the tumor is relatively small,mostly solid even low echo ( Figure 1 ), the tumor of relatively big is uneven low echo ( Figure 2 ); 4 ) The tumor showed cystic-solid mixed echo, when there was cystic change or hemorrhage and necrosis in tumor ( Figure 3 ), It is difficult to identify with other types of tumors ; (5)Some may exhibit high strength echo mass with posterior echo attenuation ( Figure 4 ) , may be associated with calcification and hemorrhage of tumor; (6)show liquid with tiny punctate echoes in a few tumors ; (7) often associated with pelvic effusion ( Figure 5 ) ; (8)CDFI shows no obvious or a small amount of blood flow signal in internal and peripheral of tumor (blood flow can only be detected in 17 cases by CDFI) ( Figure 6 ) ; (9) Can cause uterine hyperplasia or cancer or with myoma of uterus. 


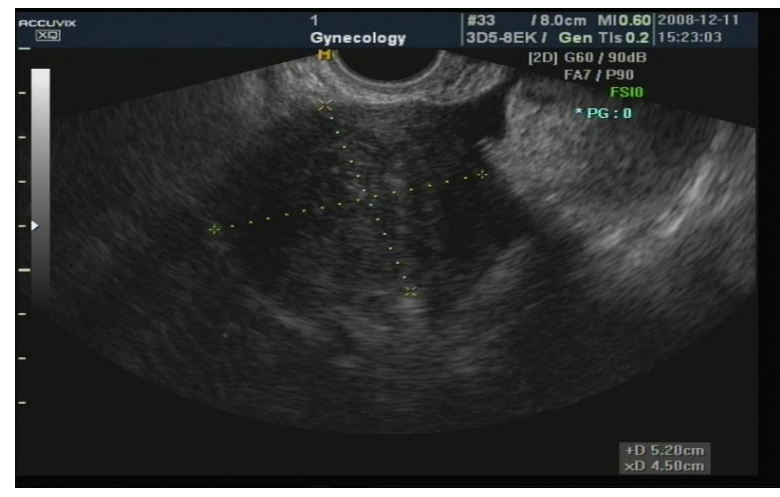

$5.2 \mathrm{~cm} \mathrm{x} 4.5 \mathrm{~cm}$ solid masses were seen in right accessories area, round, clear boundary, internal hypoechoic

Figure 1 solid masses of right accessories area

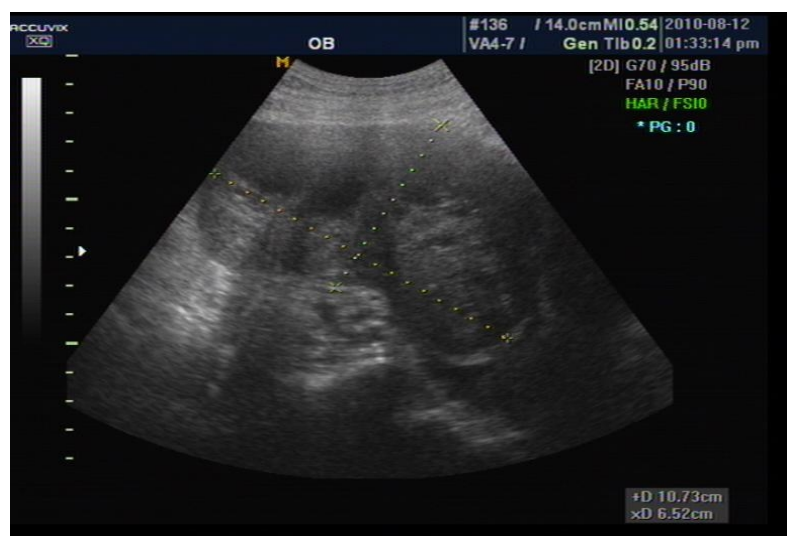

$10.7 \mathrm{~cm}$ x $6.5 \mathrm{~cm}$ cystic solid mixed tumor were seen in right accessories area, irregular shape

Figure 3 cystic solid mixed tumor of right accessories area

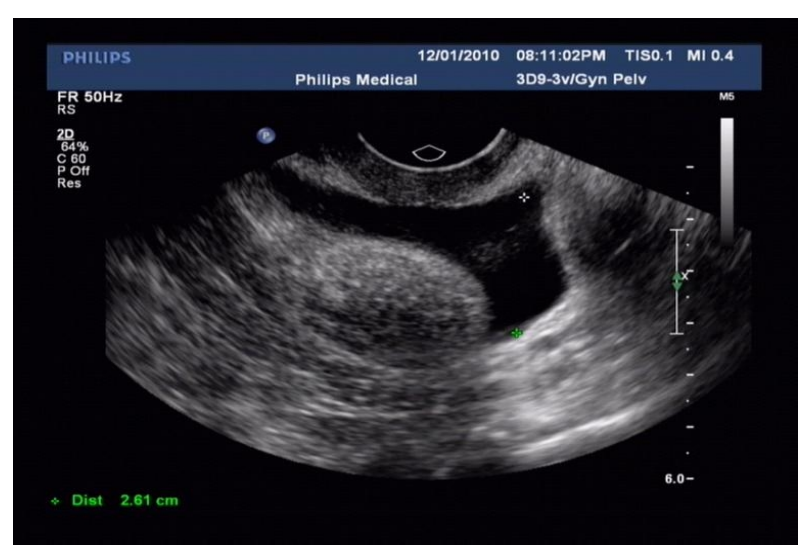

Pelvic may see free liquid of about $2.6 \mathrm{~cm}$ depth

Figure 5 Pelvic effusion

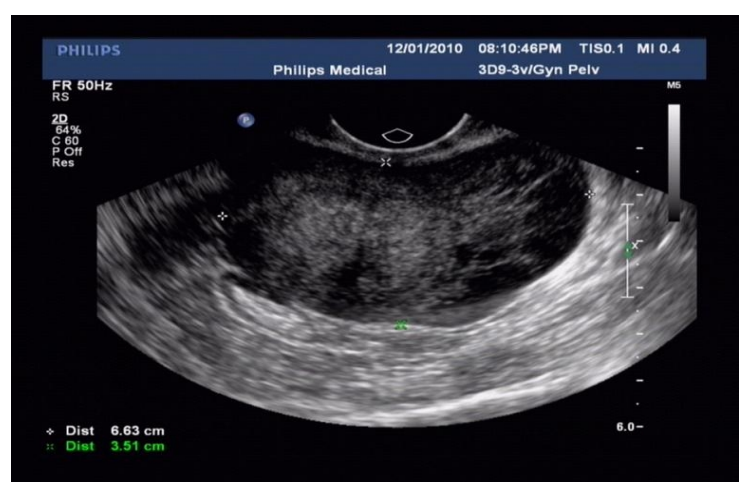

$6.6 \mathrm{~cm} \mathrm{x} 3.5 \mathrm{~cm}$ solid masses were seen behind uterine, clear boundary, uneven low echo

Figure 2 solid masses of pelvic

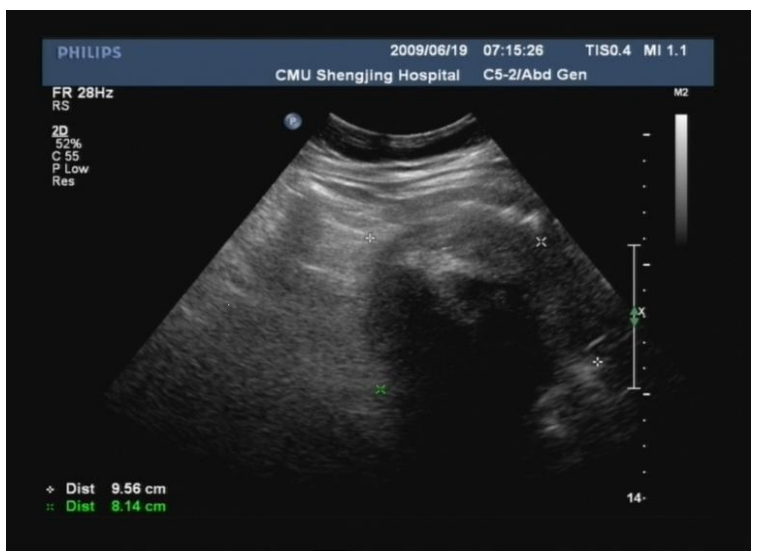

$9.6 \mathrm{~cm}$ x $8.2 \mathrm{~cm}$ middle-low mixed echo masses were seen behind uterine, with rear attenuation, fuzzy boundaries

Figure 4 solid tumor beside of the uterus

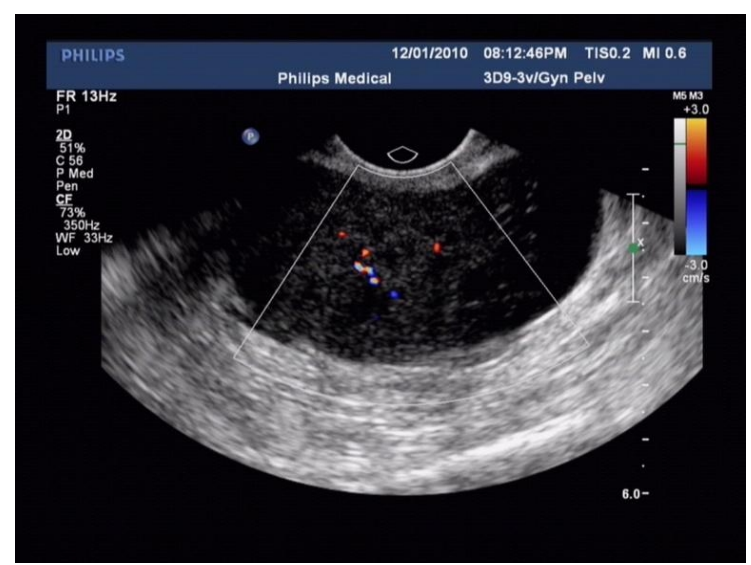

CDFImay detect flow signal

Figure 6 Color Doppler Flow Imagine(CDFI) 


\section{The clinical value of ultrasonography diagnosing ovarian thecoma}

Frequency of vaginal probe is high than frequency of abdominal probe, near pelvic organ, high resolution, image is more clear, so it can be early detect small tumors (diameter $<3 \mathrm{~cm}$ ), in demonstrating the interrelation of ovary, pelvic mass and uterus, transvaginal ultrasound has greater flexibility and superiority. But the focus areas of vaginal probe are within $10 \mathrm{~cm}$, larger tumors often fail to show panorama of picture, transabdominal ultrasound should be done, the transvaginal ultrasound and transabdominal ultrasound combined, can complement each other, foster strengths and circumvent weaknesses, make the accuracy of diagnosis more high.

\section{Limitations and missed diagnosis of ultrasonography diagnosing ovarian thecoma}

this group of 64 patients all performed ultrasonography, no one of cases can diagnose before operation, The probably reason of missed diagnosis

( 1 )The incidence rate of disease is low, insufficient attention to it; 2 )Ultrasound doctors insufficient understanding to the disease; ( 3 )A pelvic mass is larger $(>5 \mathrm{~cm})$, anatomical structure is not clear, but the elderly women's ovaries are smaller, location diagnosis of tumor is more difficult ${ }^{[5]} ; 4$ ) ovarian size of small tumor is normal, ultrasonic diagnosis is difficult, easily missed diagnosis when combined with ovarian other lesions. ( 5 ) The disease can be complicated with ascites, individual CA125 increased, has commonness with ovarian cancer, some tumors, these brings difficulty to the nature of tumor.

Prognosis of ovarian thecoma Most ovarian thecoma is benign tumor, prognosis is good , 1\%-5\% is malignant ${ }^{[2]}$.

\section{Conclusion}

Ovarian thecoma has certain ultrasnoc characteristics, the final diagnosis usually depends on pathology.

\section{Acknowledgement}

The subject is funded by the research foundation of Eastern Liaoning University, project number $2015 \mathrm{JC} 014$.

\section{Reference}

[1] Roth LM. Recent advances in the pathology and classification of ovarian sex cord-stromal tumors. Int J Gynecol Pathol. 2006 ,25(3):199-215.

[2] Nocito AL, Sarancone S, Bacchi C, et al.Ovarian thecoma: clinicopathological analysis of 50 cases. Ann Diagn Pathol. 2008 ,12(1):12-16.

[3]Stanley J,Robboy MC,Russll AP. Pathology of female genital tract[M]. HUI Yun-zhong, Translation.Peking University medical press,2005:619-622.

[4] Nemeth AJ, Patel SK. Meigs syndrome revisited. Journal of Thoracic Imaging, 2003,18(2):100-103.

[5]Szklaruk J, Tamm EP, ChoiH, et a.1 MR Imaging of Common and Uncommon Large Pelvic Masses. Radio Graphics, 2003, 23: 403 